\title{
Between a Rock and a Hard place: a Trauma-Informed Approach to Documenting the Traumatic Experiences of Tamil Refugees
}

\author{
Hilary N. Weaver ${ }^{1}$
}

Published online: 13 July 2016

(C) Springer International Publishing 2016

\begin{abstract}
Refugees and asylum seekers typically experience dislocation, persecution, and significant cultural adjustments, making them highly vulnerable populations that deserve more attention from helping professionals. As a profession grounded in human rights and committed to serving disenfranchised populations, social work is well situated to attend to the needs of refugees, asylum seekers, and other displaced populations. These populations often experience multiple forms of trauma from their own governments as well as from rebel forces. This project models a trauma-informed approach to research using an assessment tool tailored to the South Asian Tamil population. Data are presented on the traumatic experiences and related sequelae for 30 Tamils living in the USA and Canada. The majority experienced multiple traumatic events including lack of food or clean water, being displaced, lack of shelter, ill health without access to medical care, murder of a family member or someone known, being detained, and beatings. Most respondents reported dwelling on their traumatic experiences, feeling as though they were happening again, feeling hopeless, recurrent bad dreams, and having less interest in daily activities. The data presented here can inform helping professionals about the lived experiences of this population. Considerations for helping professionals working with this population are included.
\end{abstract}

Keywords Tamil · Asylum seekers · Trauma · Assessment . Refugees

Hilary N. Weaver

hweaver@buffalo.edu

1 School of Social Work, University at Buffalo, 624 Baldy Hall, Buffalo, NY 14260, USA
Since its inception, the social work profession has maintained a strong commitment to working with disenfranchised populations. Social workers have done extensive work with people who are impoverished and oppressed, yet, as a profession, social workers have done comparatively little work with refugees (Harding and Libal 2012). Refugees typically experience dislocation, persecution, and significant cultural adjustments, making them a highly vulnerable population deserving more attention from social work and other helping professions (Weaver and Burns 2001). The displacement and persecution experienced by those seeking asylum or refugee status are issues of human rights and social justice. The office of the United Nations High Commissioner for Refugees has consistently stated that forcibly displaced people are entitled to compassion and care. As affirmed by the 1951 Convention on the Status of Refugees and the subsequent 1967 Protocol, people forced to flee their country to escape persecution are entitled to recognition of their basic human rights and minimum standards of treatment (United Nations High Commissioner for Refugees 1999).

Work with refugees requires culturally appropriate and effective tools. This article describes a pilot study that used a newly modified assessment tool with a Tamil refugee population. The goals of this study were to test the instrument for internal consistency and content validity and to gather information on the experiences of this population. Tamils, a predominantly Hindu South Asian population, are a minority group from Sri Lanka. They often experience both government persecution and threats from rebel groups, thus placing them in the proverbial position of being between a rock and a hard place. The traumatic experiences of this population are described and implications for helping professionals are highlighted. 


\section{Background}

Helping professionals working with refugees, asylum seekers, and internally displaced people must recognize the ability to seek safety and live free from persecution as a basic human right and a matter of social justice. Human rights violations cause many mass exoduses. Additionally, culturally competent work with the Tamil refugee population requires an understanding of a variety of issues including the specific situation of Tamils, defining characteristics of refugee populations, and issues of trauma.

\section{Tamils and the Characteristics of Refugee Populations}

Tamils are an ethnic minority in Sri Lanka. They are a predominantly Hindu population, while the majority Sinhalese population is predominantly Buddhist. Tamils have experienced significant oppression and persecution from the Sri Lankan government and military. In turn, some Tamils fought in armed rebellion against the government and hoped to partition the country and create a separate Tamil homeland. A civil war continued for more than 2 decades punctuated by intermittent failed peace talks. The war came to define the identity of Tamil people, even after the 2009 killing of many rebel leaders led to "a cautious militarized peace without political reconciliation" (Thiranagama 2013, 21).

During the latter twentieth and early twenty-first century, young Tamil men in Sri Lanka faced a particularly difficult dilemma. They were persecuted by the government and military who often presumed that any Tamil was a rebel participating in, or supporting attacks on, the government. Rebel forces, the most famous being the Tamil Tigers or Liberation Tigers of Tamil Eelam (LTTE), often forced young men to fight as rebels whether they wanted to or not. Faced with persecution and forced into fighting by either side, many young men who were able to escape from Sri Lanka did so and sought to live safely and peaceably in other countries.

The civil war in Sri Lanka (1981-2009) led to the displacement of many Tamils. A steady flow, the majority being young men, migrated to North America and sought refugee status in Canada, while some applied for asylum in the USA. Canada was a primary destination because Toronto and Montreal both had large immigrant populations, including established Tamil communities. Additionally, Canada had a favorable record of granting refugee claims. In 1999, more people from Sri Lanka sought asylum in Canada than people from any other country (Canadian Council for Refugees 2000), but by 2010, fears that Tamils might have connections to terrorism led many Tamils to be held in detention and some to be deported (Koch 2011). Large numbers of Sri Lankan Tamils who have fled to Canada remain undocumented (George 2013).

The USA has been considerably less welcoming of Tamils seeking asylum than Canada initially was. From 1997 to 2000, the USA approved between 43 and 58 Sri Lankan asylum claims annually. In 2001, that number rose to 96 and in 2002 it was 82, but in subsequent years numbers dropped significantly (Department of Homeland Security 2016). The USA had few strong Tamil communities and was much more likely than Canada to reject a petition of a Tamil wishing to remain within its borders. Because there are few direct flights to Canada from many parts of the world, most Tamils fleeing Sri Lanka pass through the USA, even if they eventually hope to settle in Canada. Many found their way to Vive la Casa, a refugee shelter in Buffalo, NY, just a short distance from the USA-Canada border. At the time of this study, Tamils were the largest population at the Vive la Casa shelter. This population typically experienced significant postmigration stressors as many spent time in legal limbo awaiting status determinations.

Shelters like Vive la Casa view the safety of those fleeing persecution as a basic human right and a matter of social justice. They provide basic assistance such as food, clothing, and shelter as well as some legal assistance. The humanitarian assistance offered by Vive la Casa extends well beyond services offered through government channels. Since Vive la Casa serves people who have not yet acquired legal status, their residents experience uncertainty about their futures, an ongoing postmigration stressor. Under these circumstances, shelter staff members strive to provide as much stability as possible out of humanitarian concern and ideals of social justice.

Many newcomers arrive in the USA annually, but few are refugees. Refugees are narrowly defined by international law. The 1951 United Nations Convention on the Status of Refugees states that a refugee is a person who has left his or her country and cannot return due to a well-founded fear of persecution based on race, religion, nationality, membership in a particular social group, or political opinion (Martin and Yankay 2014; United Nations High Commissioner for Refugees 2014). By definition, refugees are a population of international humanitarian concern. The Convention affirms "universal respect for, and observance of human rights and fundamental freedoms for all" (Article 55, UNHCR 1999).

To qualify for refugee status, an individual must be outside his or her home country and apply to be accepted as a refugee in yet another country. Many people meet all other qualifications but are not outside the boundaries of their home country; thus they are considered to be internally displaced people (UNHCR 2014). Others who flee persecution and apply to legalize their status once in another country like the USA are asylum seekers (Martin and Yankay 2014).

Refugees, asylum seekers, and internally displaced persons typically have similar traumatic experiences but have a different legal status depending on where they reside when they apply for status in a new country. All these populations have been displaced and are not exercising a free choice to relocate. Assisting these populations is a matter of social justice. 
Significant numbers of people around the world are exposed to trauma associated with conflict, persecution, and displacement as a result of more than 200 armed conflicts since World War II (Nickerson et al. 2014). Legal definitions that distinguish between refugees, asylees, and internally displaced people are not predictors of trauma or health status (Hollifield et al. 2002). While status (i.e., refugee vs. asylee) can be important in legal terms, the label refugee is often used to describe people who flee persecution, regardless of whether they legally qualify as a refugee or asylee. The broader use of the term refugee is used throughout this article and includes those seeking asylum or refugee status.

\section{Issues of Trauma}

By definition, refugees have experienced or are at risk for persecution; thus, one of the hallmarks of the refugee experience is trauma (Hollifield et al. 2013). Indeed, many refugees have experienced multiple losses and forms of trauma. Refugees have complex trauma profiles, often including various functional limitations and comorbid conditions (Betancourt et al. 2012).

Refugees often experience multiple sources of trauma including targeted violence and torture (Slewa-Younan et al. 2015). The trauma experienced by refugees is not accidental like that experienced by survivors of natural disasters. Rather, it is the result of deliberate targeting of people, often by their own governments. This targeting may also involve torture. The United Nations Convention Against Torture and other Cruel, Inhuman or Degrading Treatment or Punishment defines torture as any act by which severe pain or suffering, whether physical or mental, is intentionally inflicted on a person for such purposes as obtaining from him or a third person information or a confession, punishing him for an act he or a third person has committed or is suspected of having committed, or intimidating or coercing him or a third person, or for any reason based on discrimination of any kind, when such pain or suffering is inflicted by or at the instigation of or with the consent or acquiescence of a public official or other person acting in an official capacity (United Nations 1984 Part 1 Article 1).

It should be noted that this Convention is only binding and enforceable on countries that have both signed and ratified it and that does not include Sri Lanka.

For refugees and asylum seekers, trauma may not end when they flee their home countries. Migration can be difficult and dangerous. One Tamil refugee described fleeing war-torn Sri Lanka and traveling through multiple countries before setting out with 150 other Tamils on a freighter bound for Canada. After 2 weeks at sea, the captain set the migrants adrift in two crowded lifeboats. They drifted for 3 days with no food or water before being spotted by fishermen off the coast of Newfoundland (Koch 2011).
For some migrants, leaving their homes may involve enlisting the help of smugglers, fleeing under dangerous circumstances, and being subjected to exploitation. The journey toward safety often involves traumatic events that extend beyond and compound the original trauma. At the time of this writing, virtually daily news coverage documents migrants fleeing combat, persecution, and extremist groups in Africa and the Middle East. These refugees try to escape on overfilled boats that often capsize before passengers arrive in conflict-free areas. This has resulted in mass drownings that compound trauma for survivors who witness the death of companions and family members and/or experience their own injuries or near-death experiences.

Tamils who fled Sri Lanka experience trauma related to both premigration and postmigration experiences (George 2013). Tamils in Canada report a PTSD rate of almost $17 \%$ compared to a national average of around $9 \%$ (Beiser et al. 2015).

Even after arrival at their destinations, refugees may continue to be exposed to traumatic events. Indeed, chronic posttraumatic stress disorder is "as much a post- as a pre-migration problem" (Beiser et al. 2015, 40). Postmigration stress can compound premigration trauma (Bentley et al. 2012). Trauma may be exacerbated by the stresses of resettlement, asylum proceedings, and threats of deportation for those without secure legal status (Betancourt et al. 2012; Gorman 2001). For example, Tamils reported that interviews by the Immigration and Refugee Board in Canada as part of the decision-making process for refugee claimants led to additional psychological distress (George 2013). Indeed, traumatic experiences are not just discrete episodes of war-related violence. Traumatic experiences often extend across premigration, migration, and resettlement periods (Betancourt et al. 2012).

While policies are subject to change, asylum seekers are often placed in administrative detention (Cleveland and Rousseau 2013). "Post-migration stressors, such as detention, tend to worsen mental health and impede recovery" (Cleveland and Rousseau, 2013, 410). Even brief detention can significantly raise levels of post-traumatic stress, depression, and anxiety among asylum seekers. This being the case, governments should consider alternatives to incarceration of asylum seekers, a vulnerable population at risk for additional psychological harm (Cleveland and Rousseau 2013).

Refugees are sometimes exposed to extreme hardships after resettlement (Betancourt et al. 2012; Tay et al. 2015). These hardships can include living in unsafe neighborhoods and being exposed to crime. Other stressors result from a xenophobic social and political environment that seems increasingly hostile to resettlement of refugees. Since 2010, Tamils seeking status in Canada have encountered a hostile climate fueled by xenophobia and fears of terrorism leading to increased detentions (Koch 2011). 
Social workers may see trauma and torture survivors as a result of conflict and human rights abuses in various parts of the world (Barrington and Shakespeare-Finch 2013). Applying a trauma-informed perspective means maximizing safety, choice, empowerment, collaboration, and trustworthiness (Fallot and Harris 2001). It is important that social workers become familiar with these principles of traumainformed care and apply them both directly with clients and in the design and implementation of services.

Government officials who work with asylum seekers and refugee claimants can also benefit from understanding and implementing the principles of trauma-informed care so as not to compound trauma. The approach used by the Immigration and Refugee Board in Canada can exacerbate trauma in Tamil claimants. Tamils seeking refugee status often reported that board members demonstrated limited "knowledge of international refugee law, ambivalence toward traumatization, ignorance regarding trauma and a lack of understanding of refugees' historical, social, cultural, and political backgrounds" (George 2013, 179). These factors adversely affect the decision-making process and increase the psychological distress of Tamil applicants for refugee status.

Memory and cognitive disorders are common complaints associated with PTSD (Carlozzi et al. 2011). Torture is often designed to confuse, give wrong information, and to create disorientation in time and space. The effectiveness of these strategies presents challenges for helping professionals and researchers seeking unequivocal or complete reports of events. When collecting data for clinical assessments or research, it is important to be careful to avoid retraumatization and demonstrate respect for survivors. Asking questions may be reminiscent of interrogation and may trigger traumatic memories (Pope 2012).

Drawing from the principles of trauma-informed care, interviewers can take steps to help refugees feel safe, exercise some level of choice, and feel empowered to collaborate in the process. Interviewers can demonstrate trustworthiness as they cultivate relationships with refugees and follow through on promises. Timing interviews so explorations of trauma take place after a relationship is established and framing the rationale and purpose for asking sensitive questions in a compelling way can help refugees feel safe and be empowered to be active partners in fostering their own healing.

\section{Project Overview}

The significant number of Tamils passing through Buffalo, NY, drew the attention of a group of helping professionals and researchers concerned about trauma and culturally appropriate service provision. The research team developed a culturally appropriate assessment tool tailored for the Tamil population for use by human service providers in the USA and Canada. As a foundation for the assessment tool, the team reviewed and modified the
Harvard Trauma Questionnaire (HTQ), a widely used tool originally developed for use with Vietnamese, Cambodian, and Lao refugees. The HTQ has been adapted, translated, and revalidated for use with a variety of refugee populations. Approval for this project was received from the Social and Behavioral Sciences Institutional Review Board at the researchers' university in 2005.

The research team received guidance from key informants to develop a Tamil-specific assessment tool. Interviews were conducted with helping professionals and researchers who had extensive experience working with Tamils as well as with Tamils already resettled in the USA and Canada. Prior to the interviews, key informants received the Harvard Trauma Questionnaire. They reviewed and critiqued each item for clarity and relevance for the Tamil population and made recommendations to ensure that item phrasing was meaningful. They also recommended new questions that reflected transit and postmigration Tamil experiences. This information was used to create the Harvard Trauma Questionnaire-Tamil version or HTQ-T (Weaver. Developing a culturally appropriate assessment tool: reflections on process considerations. (Unpublished)).

Key informants reviewed a draft of the HTQ-T and had an opportunity to suggest additional modifications prior to the pilot test. Using key informants who were Tamil or had extensive experience working with Tamils supported the face validity of the newly created instrument. Using these insiders was particularly helpful in developing questions that reflected transit and postmigration experiences of Tamils.

A trauma-informed protocol was developed to support members of the research team as well as the subjects. All members of the research team participated in regular meetings designed to debrief them after conducting interviews and analyzing data. This was done in recognition of the potential impact on the researchers of hearing and/or reading and absorbing information about the trauma and torture experienced by this population. Individualized self-care activities were encouraged and team meetings included demonstrations of strategies to promote balance and wellness.

\section{The Instrument}

The HTQ-T is an eight-page instrument consisting of three major sections plus demographic information. Section I contains 22 questions and is entitled "Things that Happened." This section has brief descriptions such as "lack of food or clean water" and "lack of shelter." Respondents are asked to check a box to indicate whether they "experienced" or "witnessed" a particular event. They can also check a box for "no." They are allowed space to elaborate on their answers, if they choose.

Section II of the HTQ-T is entitled "Personal Description" and begins with three open-ended questions asking why they left Sri Lanka, about frightening events in Sri Lanka, and traumatic events that happened in transit before arriving in 
North America. This is followed by a list of ten experiences they may have had in Canada or the USA such as, "fears of being sent home" and "language difficulties" to which they can answer "Yes" or "No." Part III, "Thoughts and Feelings," describes trauma sequelae such as "recurrent bad dreams" and "difficulty concentrating or focusing" and respondents can check boxes to indicate "A Little," "All the Time," or "Not at all."

\section{Methodology}

Tamils applying for or who had received refugee status in Canada or asylum in the USA were recruited through Vive la Casa refugee shelter and from communities in Western New York State and Ontario, Canada, with the assistance of Tamil advisors. Vive la Casa is capable of sheltering approximately 120 people at a time. Residents stay at the shelter anywhere from a few hours to several months. On any given day, there are residents from approximately 25 different countries in the shelter. Posters at Vive la Casa, recruitment by the shelter social worker, word of mouth, and snowball sampling techniques were used until the target sample number of 30 was reached. The target number was selected because it was deemed adequate for a preliminary evaluation of how subjects responded to the HTQ-T, and it could be reached with available funding.

The purposive sample was stratified to include respondents from different regions of Sri Lanka who were likely to have different experiences with trauma. Likewise, we sought a sample that was half male and half female so we could evaluate if the instrument adequately represented the experiences of women as well as men. Previously, the HTQ had been criticized as not generalizable to the experiences of women refugees (Allotey 1998; Hollifield et al. 2002). In spite of significant attempts to recruit an equal number of men and women, ultimately, the sample consisted of 23 men and 7 women. The North American Tamil population is heavily male because they are the ones most likely to flee forced participation in Sri Lanka's long-standing civil war. The sample demographics reflected the North American Tamil population. A similar project conducted with Tamil refugees consisted of 22 males and 13 females with a sample size of 35 (George 2013). By using a community sample, we strengthened generalizability and addressed a concern that instruments like the HTQ had been tested on clinical samples (Hollifield et al. 2002). Use of a community sample was an important step, but the small numbers in this pilot study limit generalizability of the findings.

Announcements about the project were made at the shelter, posted in visible areas, and distributed by Tamil advisors. People interested in participating in the project were encouraged to contact the interviewers for more information and to arrange a time for an interview. Two social workers experienced in working with refugees conducted the interviews. A male social worker interviewed male respondents, while a female social worker interviewed female respondents to maximize comfort when discussing issues of trauma, particularly questions about sexual assault. The interviewers explained the project, including that participation was entirely voluntary, would not affect subjects' ability to receive services, and they could withdraw participation at any time. Subjects were provided with a written consent form that was reviewed with them prior to the interviews. Once interviews commenced, interviewers read each question to respondents while they wrote responses on a separate questionnaire. This process was designed to insure respondents clearly understood each question while maintaining some privacy in writing their answers. To protect confidentiality, no names were recorded. The only demographic data recorded was age, gender, and marital status. Questionnaires were collected then stored in a locked location accessible only to the researchers.

Respondents ranged in age from 20 to 72 with more than half ( $n=16,53 \%$ ) being in their $20 \mathrm{~s}$ and 30 s. Fourteen respondents were single and 16 were married. Nine indicated that they were currently with their families, while 18 were not and 3 did not answer this question. In particular, women were more likely to be married and with their husbands $(n=3$, $38 \%$ ). Of the six men who were with their families, four $(67 \%)$ were in their $40 \mathrm{~s}$ or $60 \mathrm{~s}$. The other two men who indicated that they were with their families were single and in their 30s. The sample, like the population it was drawn from, was predominantly young, single men. Respondents were in the USA or Canada between 1 week and 7 years. Twelve respondents had been in North America less than a year and 17 a year or more.

This stage of the project focused on piloting the instrument to evaluate its cultural appropriateness prior to translation. Translation of an instrument that had not been determined to be culturally appropriate was deemed premature. Since the project had limited resources and we knew we would not be able to have the instrument translated into Tamil prior to the pilot test, we deliberately sought respondents fluent in English. We did not anticipate that this would be a problem since Sri Lanka was a former British colony and most Tamils were well educated and spoke English. We did, however, offer to provide interpreters for respondents who felt more comfortable speaking Tamil. The interpreters provided by the project were people fluent in both Tamil and English who were regularly used as interpreters by the shelter, although they did not have professional training as interpreters. Respondents were allowed to provide their own interpreters if they preferred. Ultimately, ten of the thirty respondents chose to use an interpreter.

Responses were recorded in English, either by the respondents or interpreters. It is possible that interpreters could influence the nature of responses. For example, a respondent 
may feel more or less comfortable disclosing information depending on their comfort with the interpreter. It may also be that use of multiple interpreters added inconsistencies to the results.

As members of the research team, the interviewers participated in all aspects of the project including developing the methodology and modifying the instrument. The team developed the interview protocol and came to consensus on the best ways to recruit participants and gather data. Immersion in the project alleviated the need for orientation or additional training to standardize how the interviewers conducted the interviews.

The interviewers were experienced social workers available to provide immediate support if subjects experienced distress during the interviews. A list of local resources was also provided in case subjects later experienced distress. Both interviewers had an ongoing relationship with the shelter where many respondents were recruited and were available for additional face-to-face counseling, had the need arisen.

\section{Piloting the Instrument}

The data from the pilot test were used to evaluate the instrument for internal consistency. This was done as a form of reliability testing. The data was analyzed using SPSS. Frequencies and correlations between instrument items were run. The internal consistency fell within the acceptable range when compared with the original HTQ. Over all, the first part of the HTQ-T, items regarding experiences with trauma, showed strong correlation ( $p<=.05$ or .01 on most items). An interesting finding was that the item, "Harmed or disrespected because you were a woman, or sexually abused," was highly correlated $(r=.773, p<.0001)$ with the item "forced isolation" and $(r=.621, p<.0001)$ with the item "land mines," but not with "loss of consciousness" $(r=.094$, $p=.648)$ or "torture" $(r=.229, p=.250)$. The alpha coefficient for the 24 symptom items was .74 indicating a correlation between all items. The instrument was found to be internally consistent.

Asking respondents to complete the assessment tool a second time is a common way of documenting reliability. We deliberately chose not to employ that method for two reasons. First, there is significant documentation that because of trauma, refugees and asylum seekers are frequently inconsistent in the information they give (Weaver et al. 2003; Wyshak 1994). Secondly, key informants emphasized that asking respondents to be interviewed or complete the same measure twice is a redundancy reminiscent of the trauma and torture experiences of many Tamil people. Although test-retest reliability is generally considered good practice in the social sciences, in this case, this procedure was likely to be of questionable utility and put the respondents at risk of retraumatization.

\section{Results}

\section{"Things That Happened"}

Respondents were asked to indicate whether they had experienced or witnessed 22 traumatic experiences common for Tamil refugees. Their responses are depicted in Table 1. The majority of respondents experienced most of the events with the most common being lack of food or clean water, being displaced, lack of shelter, other frightening situations, ill health without access to medical care, murder of a family member or someone known, and being detained. The length of detention ranged from 1 day to 1 year with the most common response being 2 months.

Being beaten (on parts of the body other than the head) was listed as personally experienced by more than half the respondents. In the following responses, efforts were made to preserve the original wording of the respondents; however, spelling and grammar were modified for clarity. When given the opportunity to explain further, half of the respondents made statements such as "they beat my forehead with a pipe" and "at the time of detention I was beaten with the butt of the gun on my shoulder and even with steel on my leg." The respondents who indicated they were tortured had similar responses such as "I was detained several times and tortured by them. They beat me with batons and cricket wicket, plastic pipes, and one time they tried to kill me. I escaped from the situation." Another stated, that he was "drowned in water, sprayed chili powder, eyes were covered with cloth, tied arms, hands, legs, beat bottom of feet." Some respondents who stated they experienced other frightening situations explained, "I am frightened of the Sri Lankan Army. Also [LTTE] tortured neighbor boys [who] were suspected, [and] dragged them into the movement." Another stated, "children taken into custody for one month and I lost my two daughters age 13 and 17 years and my house was bombed."

\section{"Personal Description"}

Respondents were asked, "Why did you leave Sri Lanka?" Twenty-nine of the 30 answered this question, but responses were often brief. For example, ten people simply stated "fear." Other responses included, "Because I have a problem with the Army and LTTE so if I stay there they are going to kill me. That's why I'm afraid to stay in Sri Lanka then I left Sri Lanka" and "I leave my country because there was no sure of my life. I had to leave due to continuous torture, discrimination by Sri Lankan government and military persons." Another stated, "life was in danger, life was no good, Army was searching for me." Eight respondents (27\%) made reference to being persecuted by the Sri Lankan government and/or the Sri Lankan Army, while two (7\%) specifically mentioned being threatened by LTTE; others simply made reference to 
Table 1 Things that happened

\begin{tabular}{llll}
\hline & Experienced & Witnessed & No \\
\hline Lack of food or clean water & $29(97 \%)$ & $7(23 \%)$ & $1(3 \%)$ \\
Ill health/no medical care & $24(80 \%)$ & $13(43 \%)$ & $1(3 \%)$ \\
Lack of shelter & $27(90 \%)$ & $10(33 \%)$ & $1(3 \%)$ \\
Being displaced in your country & $28(93 \%)$ & $10(33 \%)$ & $1(3 \%)$ \\
Detained or imprisoned & $21(70 \%)$ & $15(50 \%)$ & $4(13 \%)$ \\
Serious injury & $17(57 \%)$ & $19(63 \%)$ & $1(3 \%)$ \\
War or fighting situation & $20(67 \%)$ & $19(63 \%)$ & 0 \\
Bomb blasts & $17(57 \%)$ & $23(77 \%)$ & 0 \\
Landmines & $11(37 \%)$ & $25(83 \%)$ & $1(3 \%)$ \\
Threatened as a woman/sexual abuse & $13(43 \%)$ & $13(43 \%)$ & $6(20 \%)$ \\
Forced isolation from family or others & $19(63 \%)$ & $11(37 \%)$ & $5(17 \%)$ \\
Being close to death & $13(43 \%)$ & $22(73 \%)$ & 0 \\
Murder of family member/someone known & $22(73 \%)$ & $11(37 \%)$ & $3(10 \%)$ \\
Murder of other person & $15(50 \%)$ & $11(37 \%)$ & $6(20 \%)$ \\
Disappeared or kidnapped & $9(30 \%)$ & $19(63 \%)$ & $5(17 \%)$ \\
Drowning & $4(13 \%)$ & $11(37 \%)$ & $15(50 \%)$ \\
Suffocation & $4(13 \%)$ & $9(30 \%)$ & $16(53 \%)$ \\
Beating to the head & $11(37 \%)$ & $14(47 \%)$ & $9(30 \%)$ \\
Beating to other parts of the body & $17(57 \%)$ & $16(53 \%)$ & $4(13 \%)$ \\
Loss of consciousness & $15(50 \%)$ & $11(37 \%)$ & $6(20 \%)$ \\
Torture & $15(50 \%)$ & $17(57 \%)$ & $4(13 \%)$ \\
Other frightening situation & $25(83 \%)$ & $10(33 \%)$ & $1(3 \%)$ \\
\hline
\end{tabular}

the war, being imprisoned, or being beaten without specifying who they held responsible.

Respondents were asked, "Please tell us the most hurtful or frightening events you experienced in Sri Lanka." Twenty-eight people (93\%) responded, with 13 (43\%) referencing "bombing and gunfire", or "bombed, destruction of our belongings." More detailed answers included, "LTTE and Army both side they are fighting and bomb blasting and lot of people died", "They beat back of my body very badly while I was in jail and they tried to kill me two time and I was living in a war area in Sri Lanka." One respondent stated, "In front of my house Sri Lankan soldiers came and killed seven people and burned them and that time we were hiding and watching." Another stated, "Every time I was detained in prison I had bad experiences. I was about to be killed. Even when I was traveling I was detained from the bus, checked my luggages, identification, discrimination in front of others; therefore I had to miss the bus which I was traveling." Another stated, "Army camp near house, a yard and two boys hiding, shot by Army. Both died. Bodies taken by Army. I was suspected and had to leave after being taken to Colombo."

The third question under Personal Description began with the preface "Sometimes bad or frightening things happen in different places (for example, when refugees travel from their homes to other countries on their way to the USA or Canada). Describe any bad things that happened to you after you left Sri Lanka and before coming to either the USA or Canada." Only 11 people
(37\%) gave specific responses to this question while several others just responded "yes." Responses included "I have lots of scars," "Detained in Colombo; paid \$50,000 to the government forces," and "Initially I arrived in the U.S. I did not have any idea even I had a little money on me. I did not know how to contact a lawyer. Immediately I arrived I was handcuffed. I thought they going to deport me. I was morally lost myself."

The Personal Description section concluded with a "yes/ no" question and a list of ten common posttransit problems. "Have you had any of the following experiences since coming to the United States or Canada?" Each issue was stated by more than half the respondents. Responses to this section are presented in Table 2. The most common posttransit problems were worries about your refugee claim, fears of being sent home, delays in processing your application, worries about family back home, fewer economic opportunities, separation from family/homesickness, language difficulties, and adjusting to differences in climate, food, and culture.

\section{"Thoughts and Feelings"}

Respondents were asked to review a list of 24 common trauma sequelae or thoughts and feelings related to their experiences. They were asked to indicate "how much they bother you currently." Response choices were "A little," "All the time," or "Not at all." Most respondents experienced most trauma 
Table 2 Experiences in the USA or Canada

\begin{tabular}{lll}
\hline & Yes & No \\
\hline Worries about refugee claim & $30(100 \%)$ & 0 \\
Problems with consultant or attorney & $18(60 \%)$ & $12(40 \%)$ \\
Fewer economic opportunities & $26(87 \%)$ & $4(13 \%)$ \\
Fears of being sent home & $29(97 \%)$ & $1(3 \%)$ \\
Delays in processing your application & $28(93 \%)$ & $2(7 \%)$ \\
Separation from family/homesickness & $26(87 \%)$ & $4(13 \%)$ \\
Worries about family back home & $28(93 \%)$ & $2(7 \%)$ \\
Language difficulties & $25(83 \%)$ & $5(17 \%)$ \\
Discrimination & $19(63 \%)$ & $9(30 \%)$ \\
Adjusting to different climate, food, culture & $24(80 \%)$ & $5(17 \%)$ \\
\hline
\end{tabular}

sequelae. Responses to these questions are presented in Table 3.

\section{Discussion}

The aims of this study were to gather information on the experiences of Tamil refugees in the USA and Canada and to test the HTQ-T for internal consistency and content validity. Of particular note is the consideration the research team gave to proceeding in a trauma-informed way that not only offered protections to the research subjects but also offered support to the researchers who encountered information that could trigger their own trauma responses. The findings also add to what is known about human rights violations and traumatic reactions experienced by this particular population.

Although the sample was nonrandom and too small to support generalizing, the data collected suggests that the instrument may be valid with this population. A larger sample is needed to confirm this. Respondent direct reports are informative for social workers working with this population.

Tamil respondents experienced multiple human rights violations and traumatic events including being deprived of basic necessities such as food, clean water, shelter, and access to medical care. In Sri Lanka, respondents experienced living in the middle of a Civil War and witnessed many traumatic events. Respondents had significant exposure to death and destruction, often losing homes, belongings, and having their lives significantly disrupted. These findings align with the literature that notes refugees are likely to have experienced multiple types of trauma (Slewa-Younan et al. 2015).

Table 3 Thoughts and feelings

\begin{tabular}{|c|c|c|c|}
\hline & A little & All the time & Not at all \\
\hline Recurrent thoughts/memories of things that happened & $9(30 \%)$ & $19(63 \%)$ & $2(7 \%)$ \\
\hline Feeling the event is happening again & $9(30 \%)$ & $20(67 \%)$ & $1(3 \%)$ \\
\hline Recurrent bad dreams & $12(40 \%)$ & $15(50 \%)$ & $3(10 \%)$ \\
\hline Detached or withdrawn from people & $14(47 \%)$ & $13(43 \%)$ & $3(10 \%)$ \\
\hline Feeling like your heart is empty & $12(40 \%)$ & $17(57 \%)$ & $1(3 \%)$ \\
\hline Easily startled at loud noises/people in uniform & $14(47 \%)$ & $12(40 \%)$ & $3(10 \%)$ \\
\hline Difficulty concentrating or focusing & $16(53 \%)$ & $12(40 \%)$ & $2(7 \%)$ \\
\hline Trouble sleeping or staying in bed too much & $15(50 \%)$ & $13(43 \%)$ & $1(3 \%)$ \\
\hline Watchful or afraid of people/situations & $19(63 \%)$ & $10(33 \%)$ & $2(7 \%)$ \\
\hline Avoiding activities that remind you of frightening events & $14(47 \%)$ & $15(50 \%)$ & $1(3 \%)$ \\
\hline Sudden emotional or physical reactions when reminded of the worst events & $17(57 \%)$ & $7(23 \%)$ & $6(20 \%)$ \\
\hline Unable to remember parts of worst events & $17(57 \%)$ & $12(40 \%)$ & $1(3 \%)$ \\
\hline Less interest in daily activities & $7(23 \%)$ & $19(63 \%)$ & $4(13 \%)$ \\
\hline Hopeless & $5(17 \%)$ & $20(67 \%)$ & $3(10 \%)$ \\
\hline Feeling people don't understand what happened to you & $12(40 \%)$ & $15(50 \%)$ & $1(3 \%)$ \\
\hline Blaming yourself for things that happened & $11(37 \%)$ & $13(43 \%)$ & $4(13 \%)$ \\
\hline Feeling guilty for reaching a safe place & $19(63 \%)$ & $7(23 \%)$ & $4(13 \%)$ \\
\hline Feeling ashamed of bad things that happened to you & $17(57 \%)$ & $10(33 \%)$ & $3(10 \%)$ \\
\hline Thinking about why things happened to you & $8(27 \%)$ & $21(70 \%)$ & 0 \\
\hline Feeling as if you are losing your mind & $20(67 \%)$ & $14(47 \%)$ & $6(20 \%)$ \\
\hline Feeling others are talking about you & $6(20 \%)$ & $8(27 \%)$ & $15(50 \%)$ \\
\hline Feeling you have no one to trust & $10(33 \%)$ & $12(40 \%)$ & $8(27 \%)$ \\
\hline Sad or depressed & $10(33 \%)$ & $18(60 \%)$ & $2(7 \%)$ \\
\hline Feeling someone you trusted betrayed you & $20(67 \%)$ & $5(17 \%)$ & $4(13 \%)$ \\
\hline
\end{tabular}


Many respondents were directly targeted and experienced multiple human rights violations including detentions, beatings, and torture. There is a sense that danger permeates all aspects of their lives. Respondents have been caught in the middle of the fighting between the Sri Lankan government and various rebel factions, most notably LTTE. The Army, acting on behalf of the Sri Lankan government, was a major perpetrator of human rights violations and was responsible for many detentions and beatings. Additionally, LTTE, although they themselves are Tamils, also bear responsibility for human rights violations experienced by Tamil civilians as they tried to force them to join the rebellion. Like the findings of Beiser et al. (2015), this sample had high rates of trauma.

Some of the respondents used Section II "Personal Experiences" to give details of the human rights violations and traumatic experiences identified in Section I. As respondents answered, it is clear that many struggle with written English. Spelling of words varied considerably, particularly with the letter " $p$ " being frequently substituted for "b". Additionally, many had difficulty with English grammar. A number of statements were undecipherable and thus were left out of the analysis. The question on experiences while in transit between Sri Lanka and Canada or the USA seemed to have caused particular confusion. This question received by far the fewest responses, and some of the responses seem to report events that happened in Sri Lanka or after arriving in Canada or the USA. While it is important to examine trauma that may have occurred during transit, it is clear that this question needs to be reevaluated for effectiveness.

These experiences with the HTQ-T provide guidance for how the instrument can be refined to be most effective. For example, in the next iteration, changes can be made to find more effective ways of gathering information on traumatic experiences that occur in transit. These refinements must occur prior to translating the instrument.

These findings indicate that having respondents or their interpreters write their own answers may not be the best way of gathering information. This option was done to help protect respondents' privacy and maximize their willingness to answer while minimizing embarrassment or stigma. Those concerns would not rise to the same level if a social worker was gathering assessment data as part of the therapeutic process where the information disclosed could provide not only data on what a respondent had experienced but a platform for beginning to process those experiences and move toward healing.

Direct reports of respondents indicate that content validity and internal consistency of the instrument are generally good. The instrument seems meaningful for the Tamil context, with the possible exception of questions about in-transit experiences.

Although interpreters were offered, it was not anticipated that one third of the sample would select this option. It was important to modify the instrument for this population (thus creating the Harvard Trauma Questionnaire-Tamil or HTQ-T), but as the researchers always knew, the instrument ultimately needs to be translated to maximize its utility and insure data quality is not compromised by being only available in English.

It is clear that trauma does not end once someone has left Sri Lanka. Respondents experienced significant stressors in North America. The uncertainty and fear experienced while waiting for a final legal determination on whether they can stay in Canada or the USA compounds traumatic experiences that led them to become refugees. Trauma sequelae are common in this population and these symptoms are likely to persist, even once Tamils are no longer living in a war zone.

\section{Strengths and Limitations}

This project has numerous strengths. It is noteworthy that the sample was drawn from the community; thus the traumatic experiences are more likely to reflect those of a typical Tamil refugee rather than someone whose symptoms were heightened to the point of seeking professional assistance. The work the research team did to engage with the Tamil community led to establishing trust and gaining sanction from important Tamil gatekeepers. This enabled us to maximize getting representative data; although given the small size and nonrandom nature of the sample, this data is not generalizable.

The project expanded the knowledge base in several ways. As a project grounded in social work principles and implemented primarily by social workers, it helped move our profession forward in working with this particular vulnerable population. We took steps in applying the principles of cultural competence and insuring that Tamil-specific criteria were included in trauma assessment. Additionally, this project was initiated based on a clear need. The research team responded to a population in need of attention and used social work and research skills to close gaps in services and knowledge.

Serving refugees is more that just a clinical issue. As part of the therapeutic relationship, social workers often focus on the clinical aspects of trauma, but it is important to maintain a dual focus that also frames this as a human rights issue. The information gathered by social workers and researchers working with refugee populations can raise awareness about human rights abuses. Beyond examining trauma that happened in refugees' home countries, it is also important to call attention to postmigration trauma, particularly that encountered in settings designed to serve refugees. Increased awareness coupled with advocacy can lead to more socially just service provision and policies.

This project recognized and took steps to address the impact that exposure to refugee stories could have on members of the research team. A trauma-informed perspective was integrated throughout the project, something not typically 
considered part of data collection. Although vicarious trauma is increasingly recognized among helping professionals, this project noted that this concept is also applicable to researchers who gather or analyze refugee data. Monitoring possible vicarious traumatization and deliberate integration of self-care principles was a strength built into this research.

There are, however, limitations with this project. In spite of the concerted efforts of the researchers and advisory board members, we were not able to recruit an equal number of women and men for the pilot test. In part, this is because of a significant gender imbalance in the North American Tamil population. Additionally, it is related to the fact that Tamil men are more likely than women to have worked outside the home and feel comfortably fluent in English. Other research has found that Tamil women are less likely to integrate into North American societies than their male counterparts (Beiser et al. 2015), thus making them difficult to reach for projects like this. Some potential female respondents declined to participate because they did not feel comfortable with their English skills, in spite of the fact that interpreters were available.

An additional limitation is the small sample used in the pilot test. In the future, it will be important to evaluate the instrument using a larger sample. Given the limited resources of the project, a sample of 30 for the pilot test was deemed reasonable for this preliminary phase. Likewise, it could be considered a limitation that the instrument used was only available in English. Efforts were made to compensate for this by offering interpreters, as noted above, but this remained a significant limitation.

\section{Implications for Helping Professionals}

Helping professionals can use the HTQ-T as an assessment tool to begin to determine the types of human rights violations, persecution, and other war-related experiences that their Tamil clients have encountered. This can help identify areas for work. It may also be useful to re-administer the section of the HTQ-T on "Thoughts and Feelings" later in the therapeutic relationship to determine if there are differences in the trauma sequelae experienced by clients who have spent time working with a social worker. This may be useful in evaluating progress toward therapeutic goals.

One of the advantages of this instrument, as well as other versions of the HTQ, is that questions are phrased to gather information from the perspective of what happened to you? Rather than what is wrong with you? This is a core element of working from a trauma-informed perspective that does not pathologize or blame someone who has experienced trauma. This also fits well with a human rights perspective that recognizes refugees as people who have experienced persecution and intolerable, oppressive life circumstances not of their own making for which they cannot be blamed. Services for refugees are a matter of social justice.

This project has clear implications for practice. Helping professionals who assist refugees need to have a good understanding of trauma. Many trauma symptoms present challenges both legally and therapeutically. It is common for trauma survivors to have memory problems and tell inconsistent stories. As social workers, we expect our clients to disclose their experiences and trust us, but torture and related trauma has taught them to behave otherwise. This is an important consideration in helping refugees gain legal status and progress with their healing.

Culture is an important variable in working with refugees. Culture often shapes how trauma is expressed. Helping professionals need culturally appropriate tools and need to apply principles of cultural competence to effectively work with this population.

Helping professionals must recognize how working with refugees may affect them and their own well-being. It is important to recognize and take steps to mitigate vicarious traumatization. It is also important to recognize that processing and moving past traumatic experiences can lead to posttraumatic growth for both the client and the helping professional (Barrington and Shakespeare-Finch 2013).

In working with refugees, it is important to recognize that many trauma symptoms are actually learned methods of coping with the unspeakable events they have experienced. It is important not to pathologize survivors and see their behaviors purely in clinical terms. Rather than the problem being primarily in the individual, the problem is primarily in an environment that allows torture to take place and promotes ethnic, cultural, tribal, and religious violence. These are matters of social justice and human rights.

Helping professionals can move beyond clinical considerations and advocate for an end to situations that produce refugees. Indeed, exploring the life experiences and mental health of refugees can inform development of appropriate community-based services, shape funding priorities, and guide policy makers (Betancourt et al. 2012). Social workers can bear witness to atrocities and their impact, call for humanitarian aid, and an end to human right violations and refugeeproducing situations.

\section{Conclusion}

The millions of refugees worldwide are a population of concern to helping professionals. Often, refugees suffer trauma at the hands of both their governments and rebel forces; thus their trauma is compounded by being between a rock and a hard place. Social workers need to be concerned about human rights, from situations that produce refugees, to modifying receiving contexts, and appropriate service provision. The 
information here describes how one team of helping professionals recognized a need in a local refugee population and applied a culturally appropriate assessment tool to document the experiences of the Tamil population. This is a model for how helping professionals can be responsive to the needs of this particularly vulnerable population within the context of a trauma-informed perspective.

\section{References}

Allotey, P. (1998). Traveling with "excess baggage": health problems of refugee women in Western Australia. Women and Health, 28, 63 81.

Barrington, A. J., \& Shakespeare-Finch, J. (2013). Working with refugee survivors of torture and trauma: an opportunity for vicarious posttraumatic growth. Counseling Psychology Quarterly, 26(1), 89-105.

Beiser, M., Goodwill, A. M., Albanese, P., McShane, K., \& Kanthasamy, P. (2015). Predictors of the integration of Sri Lankan Tamil refugees in Canada: pre-migration adversity, mental health, personal attributes, and post-migration experience. International Journal of Migration, Health and Social Care, 11(1), 29-44.

Betancourt, T. S., Newnham, E. A., Layne, C. M., Kim, S., Steinberg, A. M., Ellis, H., \& Birman, D. (2012). Trauma history and psychopathology in war-affected refugee children referred for trauma-related mental health services in the United States. Journal of Traumatic Stress, 25, 682-690.

Bentley, J. A., Thoburn, J. W., Stewart, D. G., \& Boynton, L. D. (2012). Post-migration stress as a moderator between traumatic exposure and self-reported mental health symptoms in a sample of Somali refugees. Journal of Loss and Trauma, 17, 452-469.

Canadian Council for Refugees (2000). Immigration and Refugee Board, Convention Refugee Determination Division. 1999: Top Source Countries. www.web.net/ ccr/crdd99.htm. Accessed 11 June 2001.

Carlozzi, N. E., Reese-Melancon, C., \& Thomas, D. G. (2011). Memory functioning in post-traumatic stress disorder: objective findings versus subjective complaints. Stress and Health: Journal of the International Society for the Investigation of Stress, 27(3), 287-293.

Cleveland, J., \& Rousseau, C. (2013). Psychiatric symptoms associated with brief detention of adult asylum seekers in Canada. Canadian Journal of Psychiatry, 58(7), 409-416.

Department of Homeland Security. (2016). Individuals granted asylum affirmatively y region and country of origin: Fiscal years 1997 to 2006. www.dhs.gov/sites/default/files/publications/Table17D_O. xls. Accessed 14 Jan 2016.

Fallot, R. D., \& Harris, M. (2001). Using trauma theory to design service systems: new directions for mental health services. San Francisco: Jossey-Bass.

George, M. (2013). Sri Lankan Tamil refugee experiences: a qualitative analysis. International Journal of Culture and Mental Health, 6(3), $170-180$.

Gorman, W. (2001). Refugee survivors of torture: trauma and treatment. Professional psychology: Research and Practice, 32(5), 443-451.
Harding, S., \& Libal, K. (2012). Iraqi refugees and the humanitarian costs of the Iraq war: what role for social work? International Journal of Social Welfare, 21(1), 94-104.

Hollifield, M., Verbillis-Kolp, S., Farmer, B., Toolson, E. C., Woldehaimanot, T., Yamazaki, J., Holland, A., St. Clair, J., \& Soo Hoo, J. (2013). The Refugee Health Screener 15 (RHS-15): development and validation of an instrument for anxiety, depression, and PTSD in refugees. General Hospital Psychiatry, 35, 202-209.

Hollifield, M., Warner, T., Lian, N., Krakow, B., Jenkins, J., Kesler, J., Stevenson, J., \& Westermeyer, J. (2002). Measuring trauma and health status in refugees: a critical review. Journal of the American Medical Association, 288(5), 611-621.

Koch, D.G. (2011). Once, we welcomed Tamil refugees: twenty-five years later, Canada jails "boat people". The Dominion, 77, June 24. http://www.dominionpaper.ca/articles/4000.

Martin, D.C. \& Yankay, J.E. (2014). Refugees and Asylees: 2013. Office of Immigration Statistics, US Department of Homeland Security. http://www.dhs.gov. Accessed 24 Nov 14.

Nickerson, A., Liddell, B. J., Maccallum, F., Steel, Z., Silove, D., \& Bryant, R. A. (2014). Posttraumatic stress disorder and prolonged grief in refugees exposed to trauma and loss. BMC Psychiatry, 14, $1-19$.

Pope, K. S. (2012). Psychological assessment of torture survivors: essential steps, avoidable errors, and helpful resources. International Journal of Law and Psychiatry, 35, 418-426.

Slewa-Younan, S., Mond, J. M., Bussion, E., Melkonian, M., Mohammad, Y., Dover, H., Smith, M., Milosevic, D., \& Jorm, A. (2015). Psychological trauma and help seeking behaviour amongst resettled Iraqi refugees in attending English tuition classes in Australia. International Journalof Mental Health Systems, 9(5), 1-14.

Tay, A. K., Rees, S., Chen, J., Kareth, M., Lahe, S., Kitau, R., David, K., Sonoling, J., \& Silove, D. (2015). Associations of conflict-related trauma and ongoing stressors with the mental health and functioning of West Papuan refugees in Port Moresby, Papua New Guinea (PNG). PLoS One, 10(4), 1-19.

Thiranagama, S. (2013). The self at a time of war in Northern Sri Lanka. Journal of Historical Sociology, 26(1), 19-40.

United Nations. (1984). Convention Against Torture and other Cruel, Inhuman or Degrading Treatment or Punishment. http://www. hrweb.org/legal/cat.html. Accessed 22 Jan 2016.

United Nations High Commissioner for Refugees. (2014). Figures at a Glance. http://www.unhcr.org. Accessed 24 Nov 2014.

United Nations High Commissioner for Refugees. (1999). The 1951 Convention relating to the status of refugees: its relevance in contemporary context. http://www.refworld.org. Accessed 14 Jan 2016.

Weaver, H. N., \& Burns, B. J. (2001). "I shout with fear at night": understanding the traumatic experiences of refugees. Journal of Social Work, 1(2), 147-164.

Weaver, H. N., Hunt-Jackson, J., \& Burns, B. J. (2003). Asylum-seekers along the U.S.-Canada border: challenges of a vulnerable population. Journal of Immigrant and Refugee Services, 1(3/4), 81-98.

Wyshak, G. (1994). The relation between change in reports of traumatic events and symptoms of psychological distress. General Hospital Psychiatry, 16(4), 290-297. 